\title{
Development and Characterization of PEDOT:PSS/Alginate Soft Microelectrodes for Application in Neuroprosthetics
}

\author{
Laura Ferlauto', Antonio Nunzio D'Angelo', Paola Vagni', \\ Marta Jole Ildelfonsa Airaghi Leccardi', Flavio Maurizio Mor², Estelle Annick Cuttaz', \\ Marc Olivier Heuschkel${ }^{2}$, Luc Stoppini ${ }^{2}$ and Diego Ghezzi ${ }^{1 *}$ \\ ${ }^{1}$ Medtronic Chair in Neuroengineering, Center for Neuroprosthetics and Institute of Bioengineering, School of Engineering, \\ École Polytechnique Fédérale de Lausanne, Geneva, Switzerland, ${ }^{2}$ Tissue Engineering Laboratory, HEPIA, University \\ of Applied Sciences and Arts Western Switzerland (HES-SO), Geneva, Switzerland
}

\section{OPEN ACCESS}

Edited by:

Jeffrey R. Capadona

Case Western Reserve University,

United States

Reviewed by:

David Martin,

University of Delaware, United States

Jit Muthuswamy,

Arizona State University, United States

*Correspondence:

Diego Ghezz

diego.ghezzi@epfl.ch

Specialty section: This article was submitted to

Neural Technology,

a section of the journal

Frontiers in Neuroscience

Received: 26 May 2018

Accepted: 30 August 2018

Published: 19 September 2018

Citation:

Ferlauto L, D'Angelo AN, Vagni $P$,

Airaghi Leccardi MJI, Mor FM

Cuttaz EA, Heuschkel MO, Stoppini L

and Ghezzi D (2018) Development

and Characterization

of PEDOT:PSS/Alginate Soft

Microelectrodes for Application

in Neuroprosthetics.

Front. Neurosci. 12:648.

doi: 10.3389/fnins.2018.00648
Reducing the mechanical mismatch between the stiffness of a neural implant and the softness of the neural tissue is still an open challenge in neuroprosthetics. The emergence of conductive hydrogels in the last few years has considerably widened the spectrum of possibilities to tackle this issue. Nevertheless, despite the advancements in this field, further improvements in the fabrication of conductive hydrogel-based electrodes are still required. In this work, we report the fabrication of a conductive hydrogel-based microelectrode array for neural recording using a hybrid material composed of poly(3,4-ethylenedioxythiophene)-poly(styrenesulfonate), and alginate. The mechanical properties of the conductive hydrogel have been investigated using imaging techniques, while the electrode arrays have been electrochemically characterized at each fabrication step, and successfully validated both in vitro and in vivo. The presence of the conductive hydrogel, selectively electrodeposited onto the platinum microelectrodes, allowed achieving superior electrochemical characteristics, leading to a lower electrical noise during recordings. These findings represent an advancement in the design of soft conductive electrodes for neuroprosthetic applications.

Keywords: conductive hydrogel, conjugated polymers, electrode array, electrochemistry, neural recordings

\section{INTRODUCTION}

In neuroprosthetics, the realization of a performing interface between an implant and the soft tissue is of primary importance for both the preservation of the neuronal environment, and the correct functioning of the device (Lacour et al., 2016). The main approaches taken into consideration for this purpose are essentially two: the modification of the electrode surface with conductive polymers (CPs) or carbon nanotubes (CNTs) (Abidian et al., 2010; Guex et al., 2015; Charkhkar et al., 2016) and the encapsulation of the device in a hydrogel matrix (Kim et al., 2010; Heo et al., 2016). $\mathrm{CPs}$ and CNTs are versatile carbon-based materials already exploited in the fabrication of several neuroprosthetic devices (Antognazza et al., 2015; Khodagholy et al., 2015; Feyen et al., 2016; Xiang et al., 2016; Ferlauto et al., 2018). In particular, their incorporation in the active sites of recording or 
stimulating neural devices is known to lower the impedance and the stiffness of metal electrodes, to allow both electronic and ionic charge transport, and to promote cell adhesion and proliferation (Abidian et al., 2010; Harris et al., 2013; Balint et al., 2014; Martin and Malliaras, 2016; Rivnay et al., 2016; Simon et al., 2016). Despite these attractive properties, these materials still suffer from a Young's modulus far higher than the one of the tissue they are in contact with, potentially leading to important implications in terms of foreign body reaction and ultimately to the device failure (Salatino et al., 2017).

On the other hand, hydrogels are natural, or synthetic materials based on a three-dimensional network of polymer chains that can retain a large amount of water and are already used in a variety of biomedical applications, ranging from contact lenses to wound healing, and drug delivery (Buwalda et al., 2014; Caló and Khutoryanskiy, 2015; Ullah et al., 2015; Bryant and Vernerey, 2018). Their tunable mechanical properties, high water content, high porosity, and soft consistency mimic the ones of living tissues; this makes hydrogels extremely attractive for neural prostheses.

To combine the strengths of these two approaches, the exploitation of conductive hydrogels ( $\mathrm{CHs}$ ) as coatings for microelectrodes in neural implants has recently emerged as a promising strategy (Green et al., 2012, 2016; Goding et al., 2017; Staples et al., 2017; Spencer et al., 2017). A CH on the electrodes could in fact simultaneously guarantee appropriate electrochemical and mechanical properties for the interaction with the neural tissue.

To our knowledge, despite recent progress in this field, a selective micro-sized confinement of $\mathrm{CHs}$, with a thorough electrochemical characterization at each fabrication step, followed by in vitro and in vivo validation, is still missing (Kleber et al., 2017; Staples et al., 2017). The aim of this work is therefore to provide a comprehensive characterization of a $\mathrm{CH}$-based microelectrode array, consisting of a poly $(3,4-$ ethylenedioxythiophene)-poly(styrenesulfonate; PEDOT:PSS) and alginate (A) coating (called conductive alginate, CA) selectively electrodeposited on platinum $(\mathrm{Pt})$ microelectrodes to reduce the mismatch of the material properties at the electrode-tissue interface.

\section{MATERIALS AND METHODS}

\section{Electrode Arrays Used in the Study}

Pt-based microelectrode arrays (MEAs) with various geometries (linear or grid), electrode diameters (30, 100, or $400 \mu \mathrm{m}$ ), and electrode coatings (PEDOT:PSS, PEDOT:PSS/A, PEDOT:PSS/CA, and Pt Black) have been fabricated and used in this study. For structural and electrochemical characterization, planar grid MEAs (g-MEAs) with 16 electrodes $(4 \times 4)$ of $400 \mu \mathrm{m}$ in diameter and a center-to-center distance of $1 \mathrm{~mm}$ were used. Electrodes were bare Pt, or Pt-coated with PEDOT:PSS, PEDOT:PSS/A, and PEDOT:PSS/CA. For in vitro validation, two planar MEAs with eight electrodes of $30 \mu \mathrm{m}$ in diameter were used: a bottom porous MEA (p-MEA) and a top strip MEA (s-MEA). Pt electrodes were coated with Pt black or with PEDOT:PSS/CA. For in vivo validation, penetrating linear MEAs (l-MEAs) with 16 electrodes of $100 \mu \mathrm{m}$ in diameter and a center-to-center distance of $200 \mu \mathrm{m}$ were used. Electrodes were bare Pt or Pt-coated with PEDOT:PSS/CA.

\section{Electrode Array Fabrication}

Microelectrode arrays were fabricated on 4-inch silicon (Si) wafers (thickness $525 \mu \mathrm{m}$ ) with a titanium-tungsten alloy/aluminum release layer (TiW/Al, $200 \mathrm{~nm} / 1 \mu \mathrm{m}$ ). A polyimide (PI) layer (HD MicroSystems PI2611, $10 \mu \mathrm{m}$ ) was spin-coated $(1,400 \mathrm{rpm}$ for $40 \mathrm{~s})$ and then cured by a soft bake $\left(5 \mathrm{~min}\right.$ at $65^{\circ} \mathrm{C}$ and $5 \mathrm{~min}$ at $95^{\circ} \mathrm{C}$ ) followed by a hard bake $\left(1 \mathrm{~h}\right.$ at $300^{\circ} \mathrm{C}$ with nitrogen from $\left.190^{\circ} \mathrm{C}\right)$. A titanium/platinum (Ti/Pt, $5 \mathrm{~nm} / 150 \mathrm{~nm}$ ) adhesive/conductive layer was deposited by sputtering (Alliance Concept AC450). A positive photoresist (AZ1512, $2 \mu \mathrm{m}$ ) was deposited by spin-coating and soft baked at $110^{\circ} \mathrm{C}$ for 2 min before direct exposure (Heidelberg Instruments MLA150, $405 \mathrm{~nm}$ ) and development. Electrode shaping and photoresist removal were performed by chlorine dry etching (Corial 210IL) followed by oxygen plasma (500 W for $30 \mathrm{~s}$ ). MEAs were encapsulated by spin-coating an adhesion promoter (VM651, 1,000 rpm for $10 \mathrm{~s}+3,000 \mathrm{rpm}$ for $30 \mathrm{~s}$ ), spin-coating and soft baking a first PI layer (PI2611, $10 \mu \mathrm{m}$ ) followed by a second layer $(10 \mu \mathrm{m})$, and curing (soft and hard bake). Then, a Si hard mask (1 $\mu \mathrm{m})$ was deposited by sputtering (Alliance Concept AC450) and the photolithography was repeated. Dry etching (Corial 210IL) of Si and eventually PI and photoresist (respectively, chlorine and oxygen chemistries) allowed the exposure of Pt pads and electrodes. A final Si dry etching removed the remaining hard mask. An extra etching step was performed on p-MEAs to fabricate a porous substrate $(7.5 \mu \mathrm{m}$ diameters holes with a pitch of $20 \mu \mathrm{m}$ ). The MEAs were cut by a laser cutter (Optec MM200-USP) and released by $\mathrm{Al}$ anodic dissolution for $15 \mathrm{~h}$.

\section{PEDOT:PSS Coating}

An aqueous solution of 0.1 wt $\%$ 3,4-ethylenedioxythiophene (EDOT 97\%, 483028, Sigma) and 4 wt\% poly(4-styrenesulfonic acid) solution (PSS, $\mathrm{M}_{\mathrm{w}} \sim 75,000,561223$, Sigma) in deionized water was mixed by ultrasonication for $5 \mathrm{~min}$ before being filtered with $0.2 \mu \mathrm{m}$ PTFE filters (431229, Corning). The electropolymerization of PEDOT:PSS was obtained using a potentiostat (Compact Stat, Ivium). MEAs were immersed in the solution together with a silver/silver-chloride $(\mathrm{Ag} / \mathrm{AgCl})$ reference electrode and a Pt counter electrode. The potential was increased from 0.4 to $0.9 \mathrm{~V}$ in 5 steps of $0.1 \mathrm{~V}$ and $2 \mathrm{~s}$ in duration. Then, the potential was held at $0.9 \mathrm{~V}$ for $40 \mathrm{~s}$. The protocol was repeated twice and MEAs were finally cured at $65^{\circ} \mathrm{C}$ for $3 \mathrm{~h}$.

\section{Alginate and Conductive Alginate Coating}

$1 \%$ Alginate mother solution was prepared by dissolving $1 \mathrm{wt} \%$ of alginic acid from brown algae (A2033, Sigma) and $0.5 \mathrm{wt} \%$ of calcium carbonate $\left(\mathrm{CaCO}_{3}-99 \%\right.$, C5929, Sigma) in phosphatebuffered saline (PBS). The solution was then stirred and heated at 
$100^{\circ} \mathrm{C}$ for $1 \mathrm{~h}$ and all further alginate deposition solutions with different concentrations were obtained by its dilution (Cheng et al., 2011). First, an adhesion layer composed of $0.1 \mathrm{wt} \%$ EDOT and $4 \mathrm{wt} \%$ PSS in alginate deposition solution was prepared, ultrasonicated for $5 \mathrm{~min}$, and electropolymerized (as in section "PEDOT:PSS coating"). Then, alginate was electropolymerized from the alginate deposition solution with a voltage increase from 0 to $2 \mathrm{~V}$ without any intermediate step and kept constant for 2 s. MEAs were then stored in a hardening solution consisting of 1 wt $\%$ of calcium chloride $\left(\mathrm{CaCl}_{2}, \mathrm{C} 7902\right.$, Sigma) in PBS for at least $30 \mathrm{~min}$. To obtain a CA, the electropolymerization of PEDOT:PSS was then repeated twice (as in section "PEDOT:PSS Coating").

\section{Platinum Black Coating}

Platinum electrodes were coated with platinum black using a platinum solution made of: $2 \mathrm{~g} \mathrm{H}_{2} \mathrm{PtCl}_{6} \cdot \mathrm{xH}_{2} \mathrm{O}, 16 \mathrm{mg}$ $\mathrm{C}_{4} \mathrm{H}_{6} \mathrm{O}_{4} \mathrm{~Pb} \cdot 3 \mathrm{H}_{2} \mathrm{O}$, and $58 \mathrm{~g}$ of $\mathrm{H}_{2} \mathrm{O}$ (Sigma). A $700 \mathrm{mV}$ signal at $300 \mathrm{~Hz}$ was applied via a 4,284A Precision LCR Meter (Keysight Technologies) until the electrode impedance reached a magnitude of $8 \mathrm{k} \Omega$ and a phase of $-45^{\circ}$. About $10-15$ s were required to achieve sufficient plating corresponding to a black platinum coating thickness of 300 to $400 \mathrm{~nm}$.

\section{PEGDMA Coating}

The $5 \mathrm{wt} \%$ of Poly(ethylene glycol) dimethacrylate (PEGDMA, $M_{n}$ 20,000, 25406-5, Polysciences), 5 wt $\%$ of PEGDMA $\left(M_{n}\right.$ 550, 409510, Sigma), and 0.5 wt\% of 2-hydroxy-4'(2-hydroxyethoxy)-2-methylpropiophenone photoinitiator (IRGACURE 2959, 410896, Sigma) were mixed in PBS and ultrasonicated until complete dissolution. MEAs were dipped in this solution for $3 \mathrm{~s}$ and then exposed to UV light $(365 \mathrm{~nm}$, Thorlabs) for $15 \mathrm{~min}$. Afterward, MEAs were stored inside the hardening solution ( $1 \mathrm{wt} \%$ of $\mathrm{CaCl}_{2}$ in $\mathrm{PBS}$ ).

\section{Microscopic Characterization}

The thickness of the PEDOT:PSS double layer was measured using a Bruker's DektakXT Stylus Profiler. The topography and the elastic modulus surface map were taken using a Dimension Icon atomic force microscope (AFM, Bruker); measurements were performed in liquid ( 1 wt $\%$ of $\mathrm{CaCl}_{2}$ in $\mathrm{PBS}$ ), with a ScanAsyst Fluid + probe (Bruker, nominal spring constant 0.7 $\mathrm{N} \mathrm{m}^{-1}$ ). Each scan contains 512 lines of 512 data points across a $3 \mu \mathrm{m} \times 3 \mu \mathrm{m}$ surface. Images were analyzed using Gwyddion software. For each map, the average stiffness value with its SD was obtained. The variance of the stiffness was calculated as the square of the standard deviation.

\section{Electrochemistry}

Electrochemical characterizations were performed with a threeelectrode (Ag/AgCl reference electrode, Pt counter electrode) potentiostat (Compact Stat, Ivium) in PBS ( $\mathrm{pH}$ 7.4) at room temperature. Impedance spectroscopy (IS) was performed between $1 \mathrm{~Hz}$ and $1 \mathrm{MHz}$ with an AC voltage of $50 \mathrm{mV}$. Cyclic voltammetry $(\mathrm{CV})$ was obtained by sweeping a cyclic potential at a speed of $50 \mathrm{mV} \mathrm{s}^{-1}$ between -0.6 and $0.8 \mathrm{~V}$ for Pt electrodes and between -0.9 and $0.8 \mathrm{~V}$ for coated electrodes. For each electrode, the average response over 5 cycles was calculated; the anodic and cathodic charge storage capacities (CSCs) were extrapolated from the integration of the respective currents.

\section{Cell Culture}

Human neural stem cells from induced pluripotent stem cells (iPSCs) were obtained from MTI-Globalstem (GSC-4301, Thermo Fisher Scientific). They were cultured and maintained on 1:200 GelTrex LDEV-free hESC quality (A1413302, Thermo Fisher Scientific) coated flask in a proliferation medium composed of neurobasal ${ }^{\mathrm{TM}}$ medium (21103049, Thermo Fisher Scientific) supplemented with $2 \%$ B-2 $7^{\mathrm{TM}}$ supplement (17504001, Thermo Fisher Scientific), MEM non-essential amino acids (11140050, Thermo Fisher Scientific), 1\% Glutamax ${ }^{\mathrm{TM}}$ supplement (35050061, Thermo Fisher Scientific), and $20 \mathrm{ng}$ $\mathrm{ml}^{-1}$ FGF-2 (100-18B, PeproTech). For the generation of three-dimensional neurospheres (NSs), cells were detached at approximately $80 \%$ confluence with pre-warmed StemPro ${ }^{\mathrm{TM}}$ Accutase $^{\mathrm{TM}}$ (A1110501, Thermo Fisher Scientific) for 1-2 min. The single cell suspension was centrifuged for $3 \mathrm{~min}$ at $320 \mathrm{~g}$, suspended in proliferation medium and cells were counted. 500,000 cells in $3 \mathrm{ml}$ proliferating medium were added into a non-treated six-well plate. The plate was left under orbital agitation $(80 \mathrm{rpm})$ for 4 days in a cell culture incubator at $37^{\circ} \mathrm{C}$ (100\% humidity, $5 \% \mathrm{CO}_{2}$ ). $24 \mathrm{~h}$ later, the free-floating threedimensional NSs were formed by aggregation. Four days after seeding, the NS size was checked and switched to a differentiation medium composed of NeuralQ ${ }^{\mathrm{TM}}$ Basal Medium (GSM9420, GlobalStem), GS21T Supplement (GSM3100, GlobalStem), and 1\% Glutamax ${ }^{\mathrm{TM}}$ supplement (35050061, Thermo Fisher Scientific). Cultures were maintained in orbital agitation $(80 \mathrm{rpm})$ for 6 weeks. A breathable plate sealer was added in order to reduce medium evaporation. The medium was changed once a week $(3 \mathrm{ml})$. Mature NSs were then transferred to a $6 \mathrm{~mm}$ patch of pre-cut circular hydrophilic membrane supported by a six well insert (PICMORG50, Merck-Millipore) and kept in the incubator for 1 week. The use of membrane pre-cut patches facilitates the NS manipulation.

\section{Electrophysiology in vitro}

Neurospheres on membranes were transferred with forceps under a dissection microscope (Leica Microsystems) onto the center of a p-MEA device. After 1 day of recovery, NSs top surface was put in contact with an s-MEA device (coated with platinum black or conductive alginate) and electrophysiological recordings were performed using an amplifier (W2100-HS32, Multi Channel Systems) and a data acquisition system (W2100, Multi Channel Systems). The signal-to-noise ratio (SNR) was evaluated as follow: for each electrode, the noise was quantified as the standard deviation of the voltage during a $5 \mathrm{~min}$ recording, while the signal was the average peak-to-peak voltage of the spikes recorded in the same $5 \mathrm{~min}$ period. For each electrode, the SNR was computed as the signal divided by the noise. Therefore, electrodes unable to detect neural spikes have been excluded ( $n=11$ out of 16 and $n=4$ out of 21 , respectively, for s-MEA with Pt black and s-MEA-CA). 


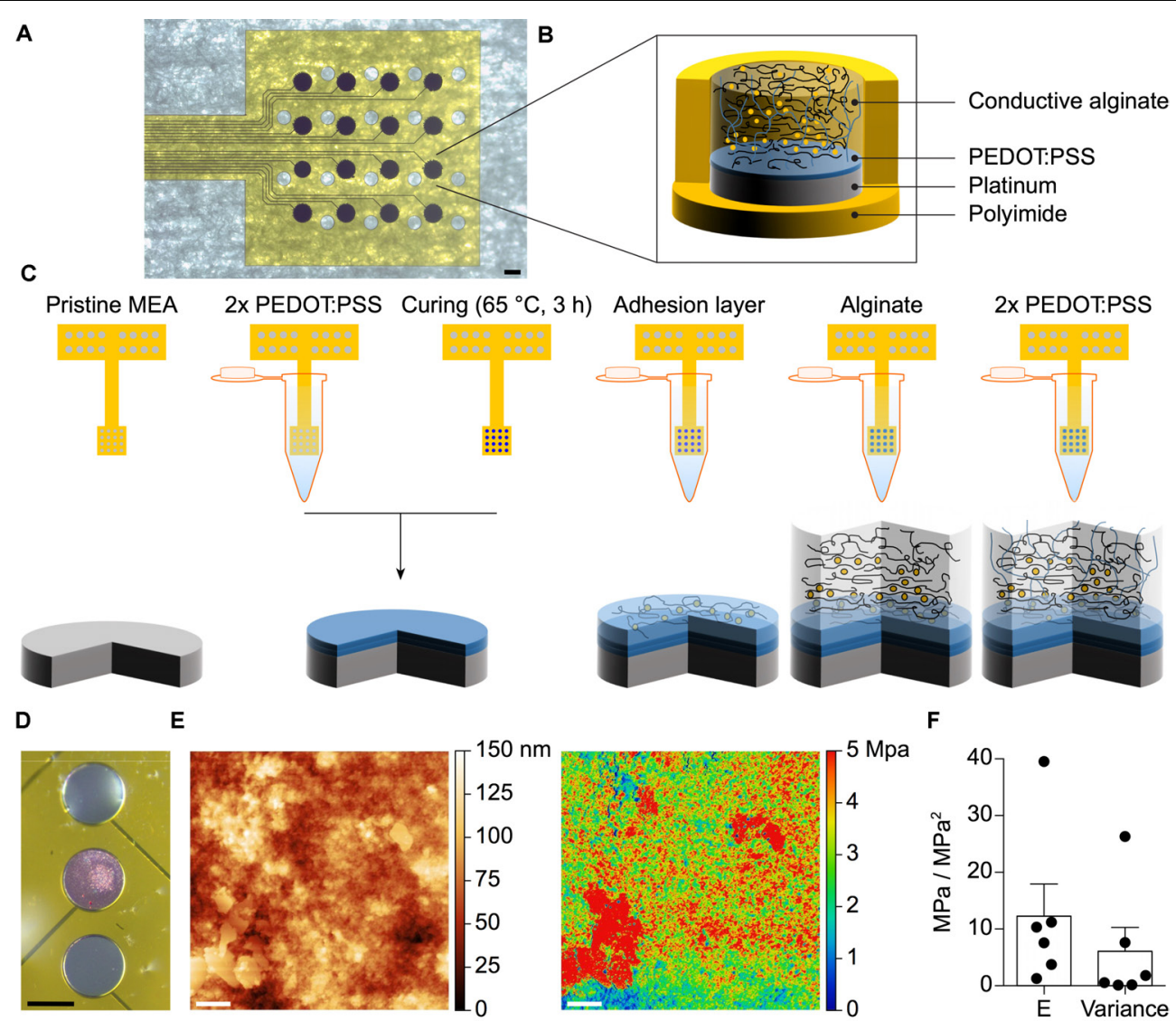

FIGURE 1 | (A) Pictures of a fabricated g-MEA with $400 \mu \mathrm{m}$ diameter electrodes. The scale bar is $400 \mu \mathrm{m}$. (B) Schematic of the soft CA-based electrode structure. (C) Step-by-step coating process of the CA-based electrodes. (D) Selective electroplating of alginate with added Rhodamine B on the central Pt electrode only. The scale bar is $150 \mu \mathrm{m}$. (E) AFM topography map of a $3 \times 3 \mu \mathrm{m}$ surface area of the CA (left) and the corresponding elastic modulus surface map (right). The scale bar is $400 \mathrm{~nm}$. (F) Quantification of the mean ( \pm SEM) stiffness and variance ( $n=6$ electrodes, 1 map per electrode).

\section{Electrophysiology in vivo}

Animal experiments were performed according to the animal authorization GE13416 issued by the Département de l'emploi, des affaires sociales et de la santé (DEAS), Direction générale de la santé of the Republique et Canton de Geneve, Switzerland. Two months old C57BL6J mice were anesthetized with isoflurane inhalation (induction $0.8-1.51 \mathrm{~min}^{-1}, 4-5 \%$; maintenance $0.8-$ $\left.1.51 \mathrm{~min}^{-1}, 1-2 \%\right)$. A $0.5 \mathrm{~mm}$ small craniotomy was opened in the correspondence of the visual cortex (identified by stereotaxic coordinates). MEAs were implanted in the cortical layers using a micromanipulator (SM-15R, Narishige). Light flashes (4 ms, 30 $c d \mathrm{~s} \mathrm{~m}^{-2}$ ) were delivered using a Ganzfeld stimulator (Biomedica Mangoni) positioned close to the contralateral eye and visually evoked cortical potentials (VEPs) were recorded and filtered $(0.1-300 \mathrm{~Hz})$.

\section{Statistical Analysis and Graphical Representation}

Statistical analysis and graphical representation were performed on Prism (GraphPad Software Inc.). The normality test (D’Agostino \& Pearson omnibus normality test) was performed in each dataset to justify the use of a parametric or nonparametric test. In each figure $p$-values were represented as: ${ }^{*} p<0.05,{ }^{* *} p<0.01,{ }^{* * *} p<0.001$, and ${ }^{* * * *} p<0.0001$. Data were always reported as mean \pm SD or \pm SEM; $n$ identifies the number of electrodes.

\section{RESULTS}

Microelectrode arrays with various geometries (linear or grid) and electrode diameters $(30,100$, or $400 \mu \mathrm{m})$ have been fabricated using PI as substrate and superstrate and Pt for the electrodes, traces, and pads (Figure 1A). Soft electrodes consisting of a first layer of PEDOT:PSS and a second layer of CA have been then electrodeposited on top of the Pt electrodes (Figures 1B,C). With a mechanical profilometer the average $( \pm \mathrm{SD}, n=12)$ thickness of the PEDOT:PSS layer has been quantified as $1.18 \pm 0.69 \mu \mathrm{m}$. The sharp confinement of the conductive alginate coating on top of the electrodes has been obtained by ionic crosslinking via electroplating (Cheng et al., 2011). This has been experimentally visualized and validated by adding Rhodamine B to the hydrogel (Figure 1D). The map of 

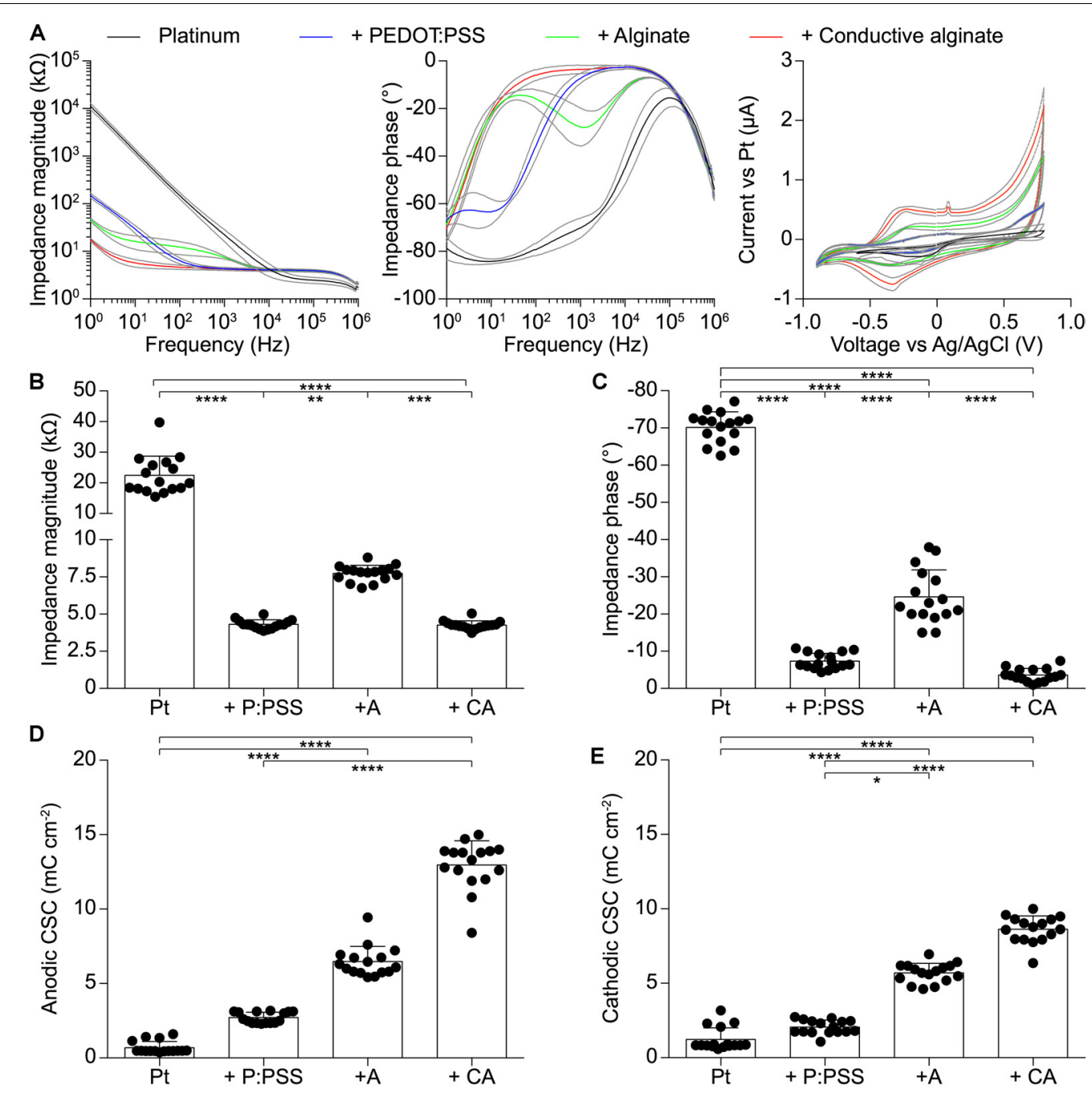

FIGURE 2 | Quantification of the electrochemical characteristics of g-MEA with 400- $\mu$ m diameter electrodes $(n=16)$ at each fabrication step. (A) IS (magnitude and phase) and CV performed at each fabrication step. Colored lines represent average values and gray lines the \pm SD. (B) Impedance magnitude at $1 \mathrm{kHz}(p<0.0001$, Kruskal-Wallis test with Dunn's multiple comparisons test). (C) Impedance phase at $1 \mathrm{kHz}$ ( $\mathrm{D}<0.0001$, one-way ANOVA with Tukey's multiple comparisons test). (D) Anodic CSC $(p<0.0001$, Kruskal-Wallis test with Dunn's multiple comparisons test). (E) Cathodic CSC ( $p<0.0001$, Kruskal-Wallis test with Dunn's multiple comparisons test). In all panels P:PSS means PEDOT:PSS.

the elastic modulus on the surface of the CA (Figure 1E) has been obtained through peak-force quantitative nanomechanical mapping atomic force microscopy (PF-QNM AFM) of the hydrated sample. The variability of the modulus values points out the presence of an interpenetrating network of a stiffer material within a softer embedding, thus revealing the co-presence of the two components of the CA. The mean ( \pm SEM) elastic modulus measured from various electrodes $(n=6)$ has been quantified in $12.29 \pm 5.67 \mathrm{MPa}$ with a mean $( \pm \mathrm{SEM})$ variance of $6.10 \pm 4.21$ $\mathrm{MPa}^{2}$ (Figure 1F).

IS and CV have been performed at each fabrication step on g-MEAs with $400 \mu \mathrm{m}$ electrodes to evaluate their electrochemical characteristics (Figure 2). In agreement with the literature (Staples et al., 2017), CA-based electrodes showed (mean \pm SD, $n=16)$ a lower impedance magnitude $(4.26 \pm 0.29 \mathrm{k} \Omega$ at
$1 \mathrm{kHz})$, a resistive behavior $\left(-3.59 \pm 1.71^{\circ}\right.$ at $\left.1 \mathrm{kHz}\right)$, and a larger charge storage capacity (CSC, anodic: $12.96 \pm 1.63 \mathrm{mC}$ $\mathrm{cm}^{-2}$, cathodic: $8.62 \pm 0.90 \mathrm{mC} \mathrm{cm}^{-2}$ ) in comparison with the other conditions tested (bare Pt, Pt-coated with PEDOT:PSS, and Pt-coated with PEDOT:PSS and pure alginate). Amongst the different concentrations of alginate within the conductive hydrogel, the lowest $(0.125 \%)$ has been chosen due to its effectiveness in constraining the gel on the electrode sites. In fact, higher gel concentrations will form a continuous layer over the entire electrode array due to the higher viscosity of the solution. From the electrochemical point of view, an improvement of impedance magnitude and phase of bare alginate coated electrodes was observed by lowering the alginate concentration, while no effect was appreciable on CA-based electrodes (Figure 3). 

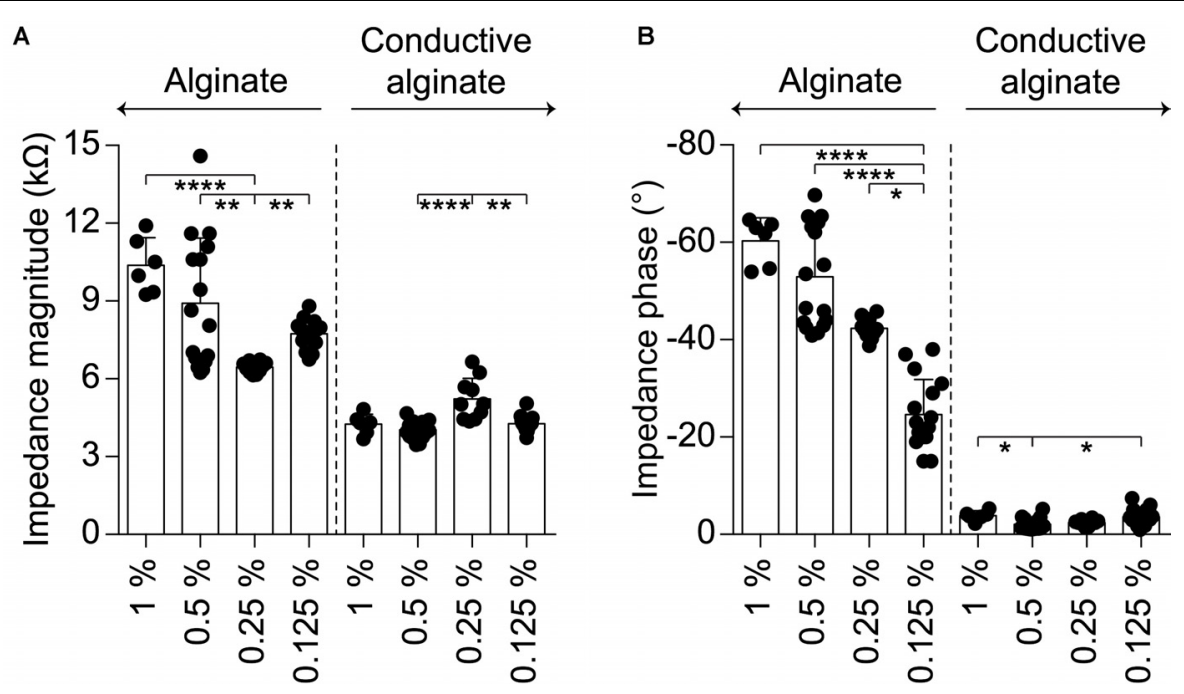

FIGURE 3 | Quantification of the electrochemical characteristics of g-MEA with 400- $\mu \mathrm{m}$ diameter electrodes at different alginate concentrations (1\%, $n=6$; 0.5\%, $n=16 ; 0.25 \%, n=10 ; 0.125 \% n=16)$; bare alginate on the left and CA on the right. (A) Impedance magnitude at $1 \mathrm{kHz}($ alginate $p<0.0001, \mathrm{CA} p<0.001$, Kruskal-Wallis test with Dunn's multiple comparisons test). (B) Impedance phase at $1 \mathrm{kHz}$ (alginate $p<0.0001$, CA $p<0.01$, one-way ANOVA with Tukey's multiple comparisons test).
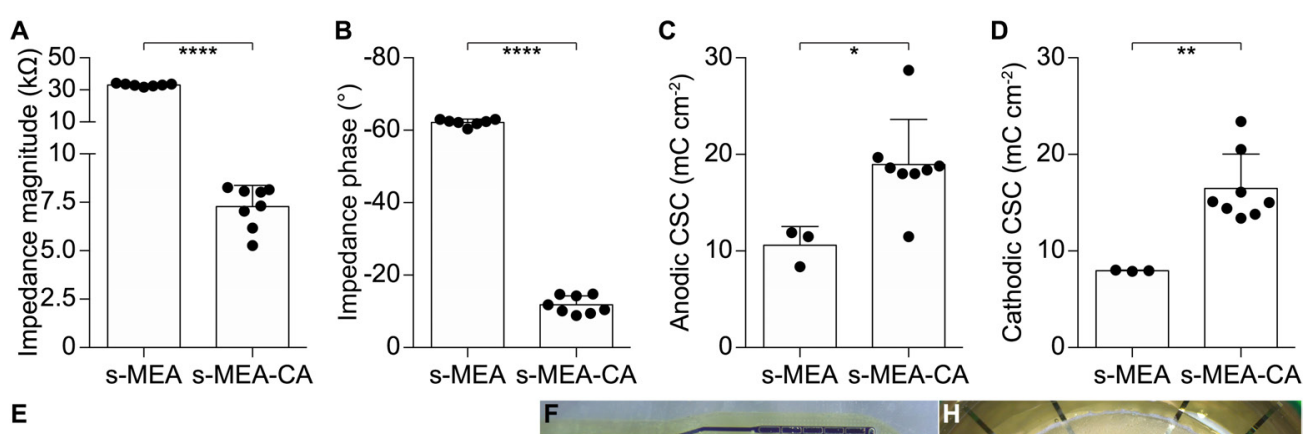

E
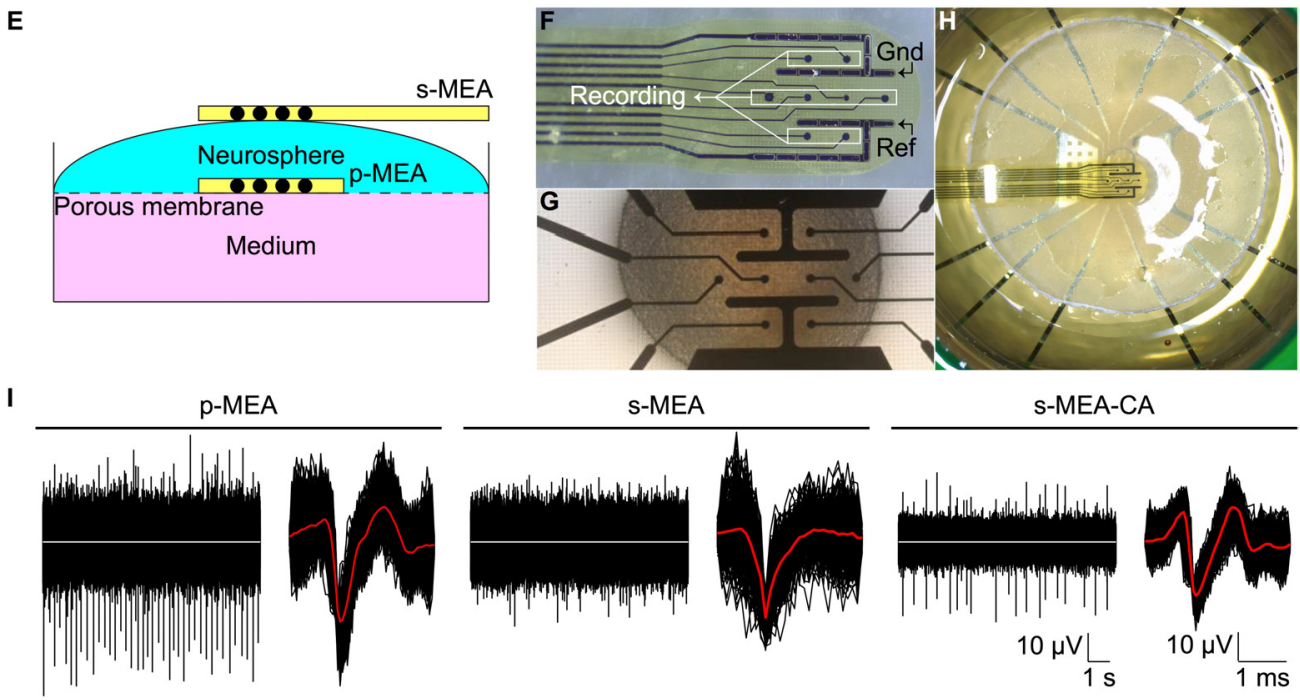

FIGURE 4 | Validation in vitro. (A-D) Quantification of the impedance magnitude at $1 \mathrm{kHz}(p<0.0001, t$-test), impedance phase at $1 \mathrm{kHz}(p<0.0001$, $t$-test), anodic CSC $(p<0.05, t$-test), cathodic CSC $(p<0.01, t$-test) of s-MEA ( $n=7$ for IS and $n=3$ for CV) and s-MEA-CA ( $n=8)$. The electrode diameter is $30 \mu m$. (E) Sketch of the recording set-up. (F) Picture of an s-MEA. (G) Picture of a p-MEA with the NS on top. (H) Picture of the set-up with p-MEA, NS, and s-MEA. (I) Representative recordings from a p-MEA, an s-MEA, and an s-MEA-CA. For each, on the left is a representative raw trace and on the right the overlay of the spike waveform from the same electrode. The white traces represent the baseline $(0 \mu \mathrm{V})$, while the red lines are the averages of the spike waveforms. 
A

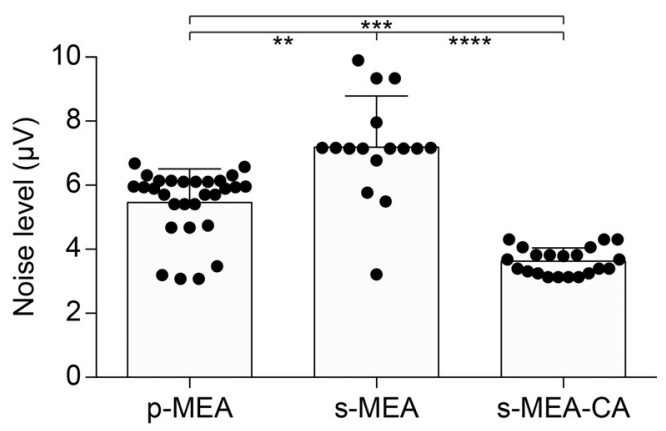

B

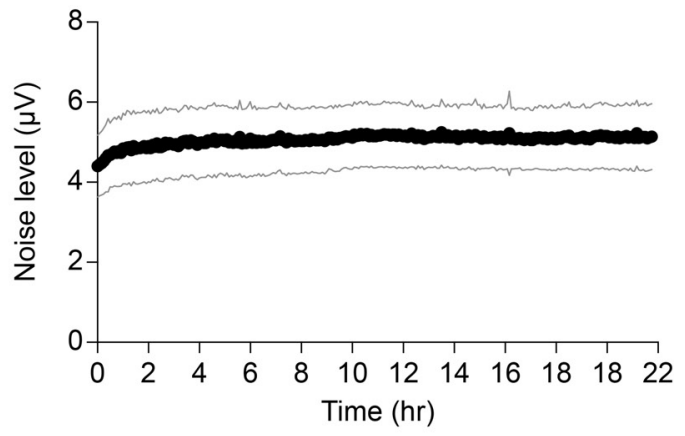

C

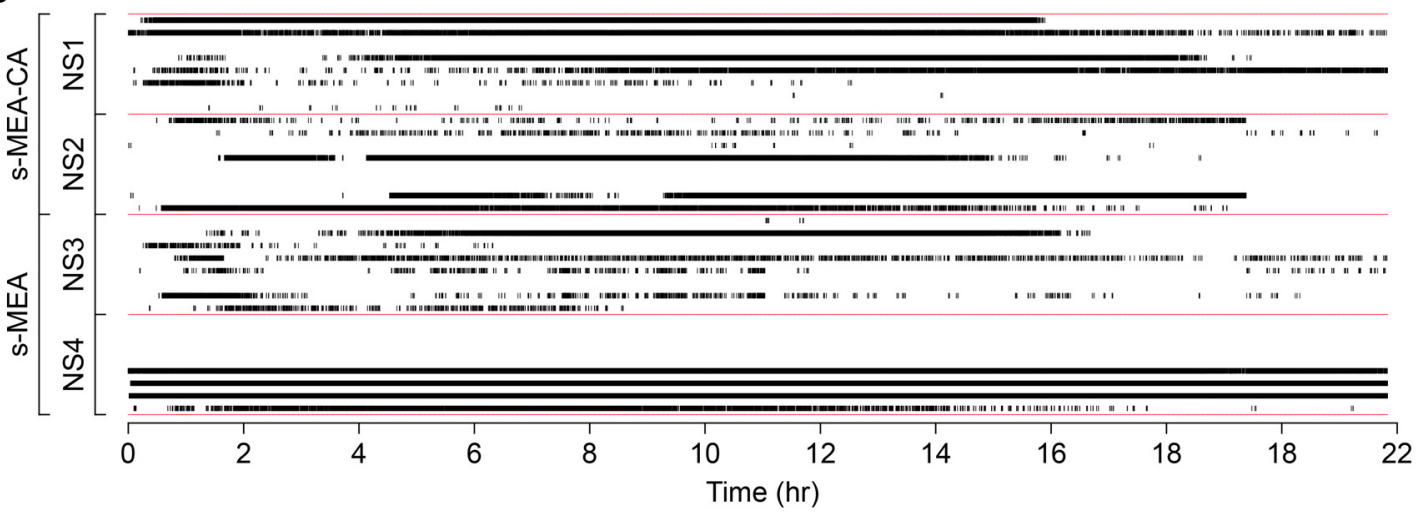

FIGURE 5 | Noise performance of CA-based electrodes. (A) Mean ( \pm SD) of the noise level between p-MEA ( $n=29)$, s-MEA $(n=16)$, and s-MEA-CA $(n=21)$; $p<0.0001$ Kruskal-Wallis test with Dunn's multiple comparisons test. (B) Mean $( \pm$ SD) noise level over the time of s-MEA-CA $(n=21)$. (C) Spike raster plot of four NSs over $22 \mathrm{~h}$ of recordings. s-MEA (two NSs) and s-MEA-CA (two NSs) have eight electrodes each.
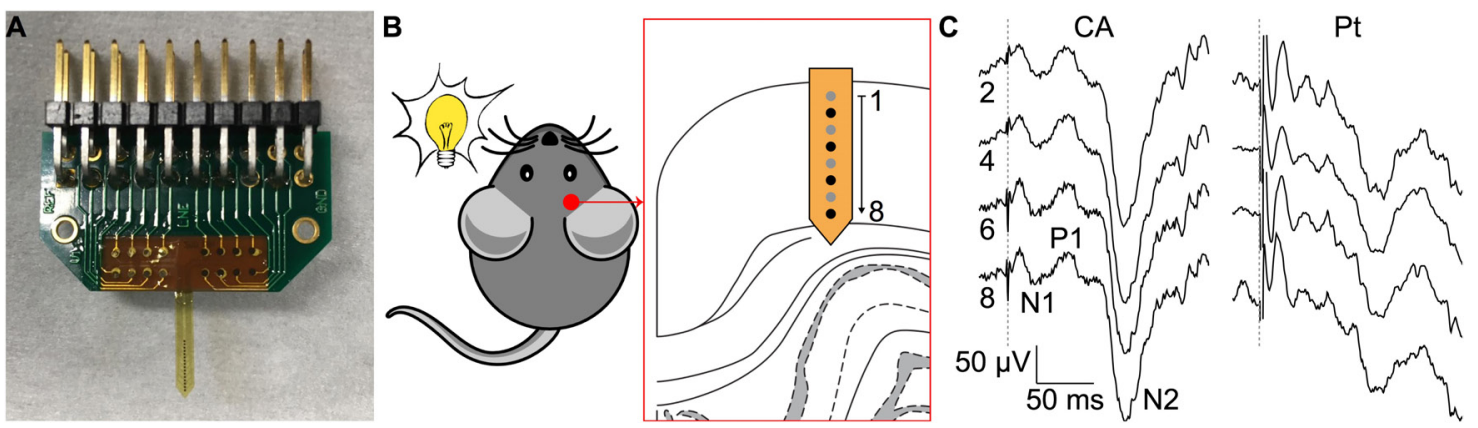

FIGURE 6 | Validation in vivo. (A) Picture of a penetrating MEA with CA electrodes. (B) Experiment sketch. (C) Representative single-sweep recordings from 4 (out of 8) CA-based electrodes (diameter $100 \mu \mathrm{m}$, black electrodes in B from an implanted array on the left and representative averaged recordings (average of 10 consecutive sweeps) from 4 (out of 8) bare Pt electrodes (diameter $100 \mu \mathrm{m}$ ) on the right. The dotted gray line represents the flash occurrence (4 ms, $30 \mathrm{~cd} \mathrm{~s} \mathrm{~m}^{-2}$ ). The main peaks of the VEP are highlighted in the trace of electrode 8.

The detailed characterization of the CA-based electrodes has been performed at $1 \mathrm{kHz}$ since this frequency is a standard reference for application in neuroprosthetics. It should be noted (Figure 2A), that at lower frequencies $(<100 \mathrm{~Hz})$, the mean impedance magnitude of PEDOT:PSS coated electrodes starts to increase, while the mean impedance magnitude of CAbased electrodes remains remarkably low till a frequency of $10 \mathrm{~Hz}$. Below $10 \mathrm{~Hz}$, it starts to increase but remains at values which are 10 times lower than those of PEDOT:PSS coated electrodes. This could be very relevant for several biomedical applications in which low-frequency signals are collected, such as for electrocardiographic and electromyographic tattoo skin sensors (Zucca et al., 2015; Wang et al., 2018).

The performance of the CA-based electrode array in recording neuronal signals has been tested in vitro on NSs formed by human neural stem cells derived from iPSCs. CA-based electrodes (30 $\mu \mathrm{m}$ in diameter) have been compared to Pt/Pt black ones, which represent the standard reference in neuronal recordings. 

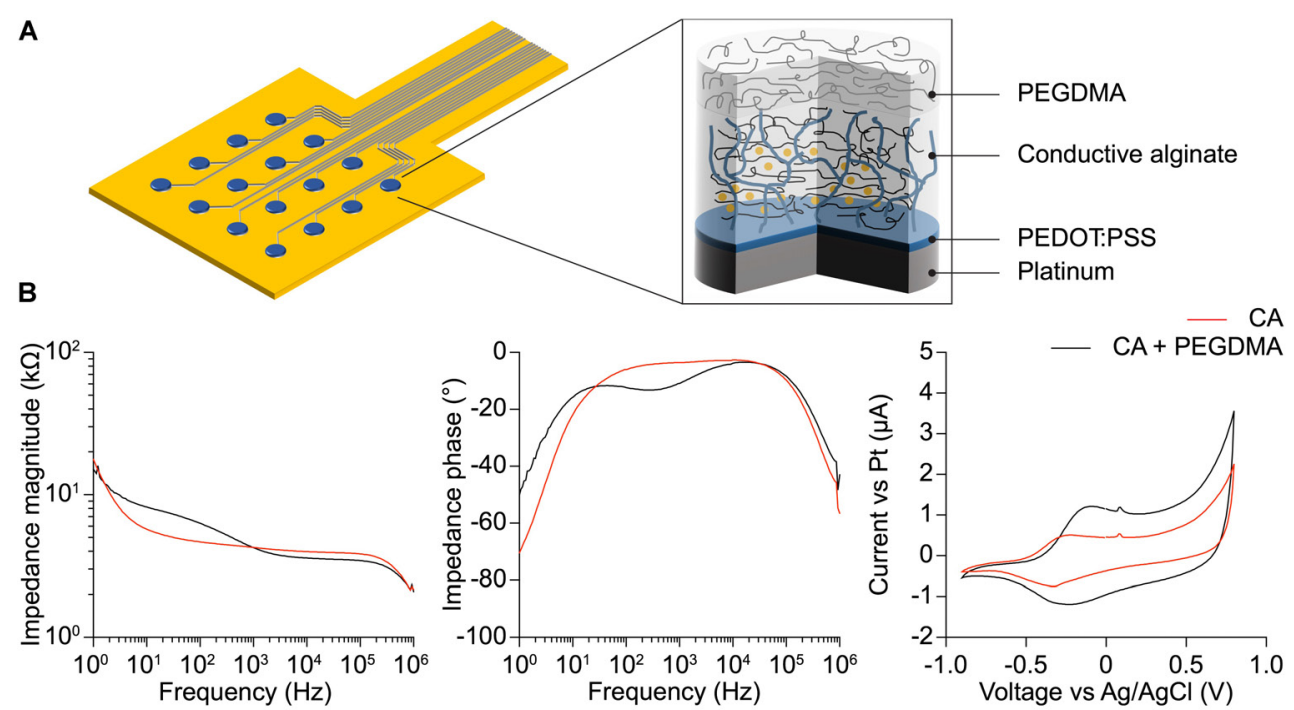

C

D

E
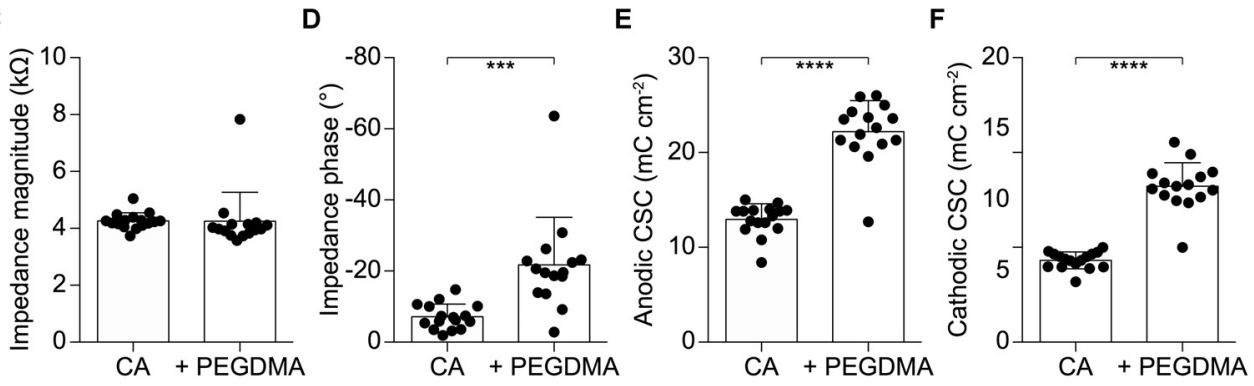

FIGURE 7 | (A) Schematic of the soft electrode structure coated with PEGDMA. (B) Mean impedance magnitude, phase, and cyclic voltammetry for CA (red) and PEGDMA (black) $400 \mu \mathrm{m}$ diameter electrodes ( $n=15)$. (C) Quantification of the impedance magnitude at $1 \mathrm{kHz}(p=0.9568, t$-test, $n=15)$. (D) Quantification of the impedance phase at $1 \mathrm{kHz}(p<0.001$, $t$-test, $n=15)$. (E) Quantification of the anodic CSC $(p<0.0001, t$-test, $n=15)$. (F) Quantification of the cathodic CSC ( $p<0.0001, t$-test, $n=15)$.

As in the previous case, the electrochemical characterization of CA-based electrodes showed (mean $\pm \mathrm{SD}, n=8$ ) a lower impedance magnitude $(7.29 \pm 1.09 \mathrm{k} \Omega$ at $1 \mathrm{kHz})$, a more resistive behavior $\left(-11.82 \pm 2.46^{\circ}\right.$ at $\left.1 \mathrm{kHz}\right)$, and an increase in the CSC values (anodic: $18.96 \pm 4.68 \mathrm{mC} \mathrm{cm}^{-2}$, cathodic: $16.46 \pm 3.57 \mathrm{mC} \mathrm{cm}^{-2}$ ) with respect to $\mathrm{Pt} / \mathrm{Pt}$ black electrodes (Figures 4A-D). For in vitro validation, four NSs have been placed onto four p-MEAs with $30 \mu \mathrm{m} \mathrm{Pt} / \mathrm{Pt}$ black electrodes, used as a reference for tissue viability. The top side of each NS has been contacted with an s-MEA embedding either $30 \mu \mathrm{m}$ $\mathrm{Pt} / \mathrm{Pt}$ black (s-MEA) or $30 \mu \mathrm{m}$ CA-based (s-MEA-CA) electrodes (Figures 4E-H). Both p-MEA and s-MEA showed a noise level larger than s-MEA-CA (Figure 4I), that has been quantified as the standard deviation of the voltage recordings. In agreement with IS (Figure 4A), CA-based electrodes (s-MEA-CA) showed a better noise level (Figure 5A) compared to $\mathrm{Pt} / \mathrm{Pt}$ black electrodes (p-MEA and s-MEA). Since NSs are seeded on top of the p-MEA, they had a stronger adhesion to the tissue, which translated into the detection of spikes with a higher peak-to-peak voltage with respect to any s-MEA (with or without CA). On the contrary, the s-MEA suffered from a weak contact to the NSs due to their spherical surface and the flexibility of the PI strip; this resulted in the detection of spikes with a lower peak-to-peak voltage (Figure 4I). However, regardless of the weaker contact, the s-MEA-CA devices presented a lower noise level in comparison with the s-MEA and p-MEA (Figure 5A), which turned into a better SNR with respect to the equivalent condition with s-MEAs: mean SNRs $( \pm \mathrm{SD})$ are $6.75 \pm 0.87(n=5)$ and $8.20 \pm 1.35$ $(n=17)$, respectively, for s-MEA with Pt black and s-MEACA $(p<0.05$, unpaired $t$-test). Moreover, the noise level of s-MEA-CA devices was not affected over a time-period of 22 h (Figure 5B), in which neuronal spikes have been detected (Figure 5C). The frequency of the detected spikes decreases after about $16 \mathrm{~h}$ of recordings. This effect has not been associated with a deterioration of the CA-based electrodes since both electrode types (s-MEA and s-MEA-CA) showed a similar reduction. On the contrary, it could be owed to a reduction of the NS viability.

The ability of CA-based electrodes to detect neuronal activity has also been proven in vivo. Penetrating CA-based MEAs with linear geometry and electrode diameters of $100 \mu \mathrm{m}$ have been implanted in the visual cortex of mice (Figure 6A). Due to the thin thickness of the mouse visual cortex, only the first eight electrodes of the probe (16 in total) have been inserted over the entire cortical thickness (Figure 6B). 
VEPs have been successfully recorded upon light stimulation of the contralateral eye (Figure $6 \mathrm{C}$, left). Because of the low impedance of the CA electrodes, individual responses to a single flash highlighted all main components (positive and negative peaks, respectively, $\mathrm{P}$ and $\mathrm{N}$ ) of the cortical VEP, without synchronous averaging. As a qualitative comparison, the cortical VEP recorded with bare Pt electrodes (diameter of $100 \mu \mathrm{m}$ ) upon synchronous averaging of 10 consecutive responses have been shown (Figure 6C, right). Qualitatively, it is visible that CA-based electrodes have better performances even without synchronous averaging. Lastly, taking into consideration the possibility of the alginate to dissolve with time during chronic implantation (Lee and Mooney, 2012), we also verified that a protective coating of the probe with Poly(ethylene glycol) dimethacrylate (PEGDMA), created by dip-coating, would not alter the electrochemical properties of the CA-based electrodes (Figure 7).

\section{CONCLUSION}

The use of CA as a soft coating of MEAs is an attractive strategy to both reduce the mechanical mismatch at the electrodetissue interface and improve the electrochemical properties of microelectrodes (Green et al., 2012, 2016; Goding et al., 2017). Our results showed a selective and sharp micro-sized confinement of CA onto the metallic electrodes of MEAs, which is one of the open challenges in the field. In addition, we provided a comprehensive and complete characterization of CA-based MEAs via electrochemical, mechanical, and electrophysiological analyses. With respect to $\mathrm{Pt}$ and $\mathrm{Pt} / \mathrm{Pt}$ black electrodes, which are the standard materials employed in clinical devices, the soft CA-based microelectrodes presented in this work demonstrated lower impedance magnitude, higher CSCs, and a more resistive behavior. This turns into an improved SNR during neuronal recordings. These results represent an important advancement

\section{REFERENCES}

Abidian, M., Corey, J., Kipke, D., and Martin, D. (2010). Conductingpolymer nanotubes improve electrical properties, mechanical adhesion, neural attachment, and neurite outgrowth of neural electrodes. Small 6, 421-429. doi: 10.1002/smll.200901868

Antognazza, M. R., Martino, N., Ghezzi, D., Feyen, P., Colombo, E., Endeman, D., et al. (2015). Shedding light on living cells. Adv. Mater. 27, 7662-7669. doi: 10.1002/adma.201403513

Balint, R., Cassidy, N., and Cartmell, S. (2014). Conductive polymers: towards a smart biomaterial for tissue engineering. Acta Biomater. 10, 2341-2353. doi: 10.1016/j.actbio.2014.02.015

Bryant, S., and Vernerey, F. (2018). Programmable hydrogels for cell encapsulation and neo-tissue growth to enable personalized tissue engineering. Adv. Healthc. Mater. 7:1700605. doi: 10.1002/adhm.201700605

Buwalda, S., Boere, K., Dijkstra, P., Feijen, J., Vermonden, T., and Hennink, W. (2014). Hydrogels in a historical perspective: from simple networks to smart materials. J. Control Release 190, 254-273. doi: 10.1016/j.jconrel.2014. 03.052

Caló, E., and Khutoryanskiy, V. (2015). Biomedical applications of hydrogels: a review of patents and commercial products. Eur. Polym. J. 65, 252-267.

Charkhkar, H., Knaack, G., McHail, D., Mandal, H., Peixoto, N., Rubinson, J., et al. (2016). Chronic intracortical neural recordings using microelectrode arrays coated with PEDOT-TFB. Acta Biomater. 32, 57-67. for the fabrication of performing neuroprosthetic devices able to reduce the mechanical and electrical mismatch at the electrodetissue interface.

\section{DATA AVAILABILITY STATEMENT}

The authors declare that all the data supporting the findings of the study are available in this article. Access to our raw data can be obtained from the corresponding author upon reasonable request.

\section{AUTHOR CONTRIBUTIONS}

LF designed, fabricated, and tested the MEAs, co-supervised the study, and wrote the manuscript. AD optimized the conductive hydrogel coating and performed the electrochemical characterizations. PV performed the in vivo electrophysiology. MA fabricated the MEAs and optimized the electrodeposition of the PEDOT:PSS. FM performed and analyzed the in vitro electrophysiology. EC contributed to the electrochemical characterization. MH fabricated the Pt-black MEAs. LS designed the in vitro assay. DG designed and led the study, validate the data analysis, and wrote the manuscript. All the authors read and accepted the manuscript.

\section{FUNDING}

This work was supported by École Polytechnique Fédérale de Lausanne, Medtronic, and European Commission (EU project 701632). The Tissue Engineering Laboratory received support from the Wyss Center for Bio- and Neuro-Engineering, Geneva, and HES-SO for the cell culture assay.

Cheng, Y., Luo, X., Betz, J., Payne, G., Bentley, W., and Rubloff, G. (2011). Mechanism of anodic electrodeposition of calcium alginate. Soft Matter 7, 5677-5684.

Ferlauto, L., Leccardi, M., Chenais, N., Gilliéron, S., Vagni, P., Bevilacqua, M., et al. (2018). Design and validation of a foldable and photovoltaic wide-field epiretinal prosthesis. Nat. Commun. 9:992. doi: 10.1038/s41467-018-03386-7

Feyen, P., Colombo, E., Endeman, D., Nova, M., Laudato, L., Martino, N., et al. (2016). Light-evoked hyperpolarization and silencing of neurons by conjugated polymers. Sci. Rep. 6:22718. doi: 10.1038/srep22718

Goding, J., Gilmour, A., Martens, P., Poole-Warren, L., and Green, R. (2017). Interpenetrating conducting hydrogel materials for neural interfacing electrodes. Adv. Healthc. Mater. 6:1601177. doi: 10.1002/adhm.201601177

Green, R., Baek, S., Poole-Warren, L., and Martens, P. (2016). Conducting polymer-hydrogels for medical electrode applications. Sci. Technol. Adv. Mater. 11:014107.

Green, R., Hassarati, R., Goding, J., Baek, S., Lovell, N., Martens, P., et al. (2012). Conductive hydrogels: mechanically robust hybrids for use as biomaterials. Macromol. Biosci. 12, 494-501. doi: 10.1002/mabi.201100490

Guex, A. A. A., Vachicouras, N., Hight, A. E., Brown, M. C., Lee, D. J., and Lacour, S. P. P. (2015). Conducting polymer electrodes for auditory brainstem implants. J. Mater. Chem. B Mater. Biol. Med. 3, 5021-5027.

Harris, A., Morgan, S., Chen, J., Kapsa, R., Wallace, G., and Paolini, A. (2013). Conducting polymer coated neural recording electrodes. J. Neural Eng. 10:016004. doi: 10.1088/1741-2560/10/1/016004 
Heo, D., Song, S.-J., Kim, H.-J., Lee, Y., Ko, W.-K., Lee, S., et al. (2016). Multifunctional hydrogel coatings on the surface of neural cuff electrode for improving electrode-nerve tissue interfaces. Acta Biomater. 39, 25-33. doi: 10.1016/j.actbio.2016.05.009

Khodagholy, D., Gelinas, J. N., Thesen, T., Doyle, W., Devinsky, O., Malliaras, G. G., et al. (2015). NeuroGrid: recording action potentials from the surface of the brain. Nat. Neurosci. 18, 310-315. doi: 10.1038/ nn. 3905

Kim, D.-H., Wiler, J., Anderson, D., Kipke, D., and Martin, D. (2010). Conducting polymers on hydrogel-coated neural electrode provide sensitive neural recordings in auditory cortex. Acta Biomater. 6, 57-62. doi: 10.1016/j. actbio.2009.07.034

Kleber, C., Bruns, M., Lienkamp, K., Rühe, J., and Asplund, M. (2017). An interpenetrating, microstructurable and covalently attached conducting polymer hydrogel for neural interfaces. Acta Biomater. 58, 365-375. doi: 10. 1016/j.actbio.2017.05.056

Lacour, S., Courtine, G., and Guck, J. (2016). Materials and technologies for soft implantable neuroprostheses. Nat. Rev. Mater. 1:16063.

Lee, K., and Mooney, D. (2012). Alginate: properties and biomedical applications. Prog. Polym. Sci. 37, 106-126.

Martin, D. C., and Malliaras, G. G. (2016). Interfacing electronic and ionic charge transport in bioelectronics. ChemElectroChem 3, 686-688.

Rivnay, J., Inal, S., Collins, B. A., Sessolo, M., Stavrinidou, E., Strakosas, X., et al. (2016). Structural control of mixed ionic and electronic transport in conducting polymers. Nat. Commun. 7:11287. doi: 10.1038/ncomms1 1287

Salatino, J., Ludwig, K., Kozai, T., and Purcell, E. (2017). Glial responses to implanted electrodes in the brain. Nat. Biomed. Eng. 1, 862-877.

Simon, D. T., Gabrielsson, E. O., Tybrandt, K., and Berggren, M. (2016). Organic bioelectronics: bridging the signaling gap between biology and technology. Chem. Rev. 116, 13009-13041.
Spencer, K., Sy, J., Ramadi, K., Graybiel, A., Langer, R., and Cima, M. (2017). Characterization of mechanically matched hydrogel coatings to improve the biocompatibility of neural implants. Sci. Rep. 7:1952. doi: 10.1038/s41598-01702107-2

Staples, N., Goding, J., Gilmour, A., Aristovich, K., Byrnes-Preston, P., Holder, D., et al. (2017). Conductive hydrogel electrodes for delivery of long-term high frequency pulses. Front. Neurosci. 11:748. doi: 10.3389/fnins.2017.00748

Ullah, F., Othman, M., Javed, F., Ahmad, Z., and Akil, H. (2015). Classification, processing and application of hydrogels: a review. Mater. Sci. Eng. C 57, 414-433. doi: 10.1016/j.msec.2015.07.053

Wang, Y., Qiu, Y., Ameri, S., Jang, H., Dai, Z., Huang, Y., et al. (2018). Low-cost, $\mu \mathrm{m}$-thick, tape-free electronic tattoo sensors with minimized motion and sweat artifacts. NPJ Flexible Electron. 2:6.

Xiang, Z., Yen, S., Sheshadri, S., Wang, J., Lee, S., Liu, Y., et al. (2016). Progress of flexible electronics in neural interfacing - a self-adaptive non-invasive neural ribbon electrode for small nerves recording. Adv. Mater. 28, 4472-4479. doi: 10.1002/adma.201503423

Zucca, A., Cipriani, C., Sudha, Tarantino, S., Ricci, D., Mattoli, V., et al. (2015). Tattoo conductive polymer nanosheets for skin-contact applications. Adv. Healthc. Mater. 4, 983-990. doi: 10.1002/adhm.201400761

Conflict of Interest Statement: The authors declare that the research was conducted in the absence of any commercial or financial relationships that could be construed as a potential conflict of interest.

Copyright (c) 2018 Ferlauto, D’Angelo, Vagni, Airaghi Leccardi, Mor, Cuttaz, Heuschkel, Stoppini and Ghezzi. This is an open-access article distributed under the terms of the Creative Commons Attribution License (CC BY). The use, distribution or reproduction in other forums is permitted, provided the original author(s) and the copyright owner(s) are credited and that the original publication in this journal is cited, in accordance with accepted academic practice. No use, distribution or reproduction is permitted which does not comply with these terms. 